\title{
Laparoscopic Repair of Congenital Lumbar Hernia in a 4 Week Old Infant
}

\author{
Faraz A Khan, Saleem Islam*
}

Division of Pediatric Surgery, Department of Surgery, University of Florida College of Medicine.1600 SW Archer Rd. P.O. Box 10019, Gainesville, FL USA

\author{
Correspondence*: Saleem Islam, Division of Pediatric Surgery, Department of Surgery, University of Florida College of Medicine. 1600 SW \\ Archer Rd. P.O. Box 10019, Gainesville, FL USA. \\ E-mail: saleem.islam@surgery.ufl.edu (C) 2018, Khan et al, \\ Submitted: 01-10-2017 \\ Accepted: 11-11-2017 \\ Conflict of Interest: None \\ Source of Support: Nil
}

This is an open-access article distributed under the terms of the Creative Commons Attribution License, which permits unrestricted use, distribution, and reproduction in any medium, provided the original work is properly cited.

\section{DEAR SIR}

Hernias of the lumbar region are a rare entity, with approximately 300 patients reported in the literature, and vary in etiology and anatomic location of origin.[1] The vast majority of lumbar hernias are acquired in etiology following traumatic injury or sugical interventions.[2] Moreover, congenital lumbar hernias (CLH) are an even rarer entity representing approximately $20 \%$ of all lumbar hernias reported.

CLHs are frequently associated with a multitude of other congenital anomalies of the trunk, musculature, ribs and vertebrae. These constellation of findings were initially described by Touloukian as the lumbocostovertebral syndrome in 1972 and since then approximately 50 cases have been reported in the english literature.[3]

A variety of techniques have been utilized to repair lumbar hernias in adults ranging from open primary tissue repair, local flaps, open and laparoscopic mesh repairs. [1, 2] CLH in children have been repaired using the traditional open approach with mesh reinforcement performed as dictated by the size of the defect.[4] Over the last several decades, a number of laparoscopic techniques have been utilized in the treatment of common pediatric surgical conditions. We report a CLH repaired laparoscopically in a pediatric age patient. Patient was a 37 week gestational age female infant, seen at day of life 10 in the pediatric surgical clinic with a chief complaint of a bulge in the right lumbar region. The patient was small for gestatation age with a weight of $2.51 \mathrm{~kg}$, length $42.5 \mathrm{~cm}$ and weight for age $z$-score -0.90

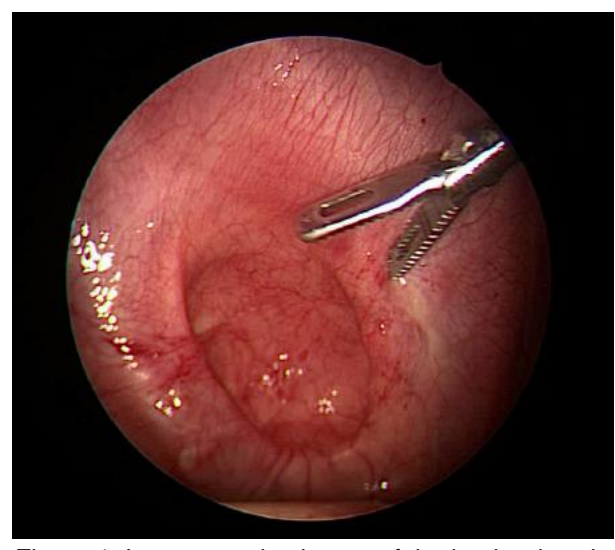

Figure 1: Intra-operative image of the lumbar hernia

Her examination was otherwise unremarkable except for the presence of $3 \times 3 \mathrm{~cm}$ hernia defect located in the inferior lumbar triangle (Petit's hernia). The patient's family was offered and subsequently underwent elective laparoscopic lumbar hernia repair. The repair was performed using a 5mm MiniStepTM port (Covidien; Dublin, Ireland) placed through the umbilicus for insufflation and insertion of laparoscope and two $3 \mathrm{~mm}$ stab incisions for laparoscopic instruments. The hernia was readily identified (Fig.1) and the hernia sac was excised following which the fascial edges were reapproximated (Fig.2) with intra-corporeal interrupted 2-0 Ethibond-ExcelTM 
sutures (Ethicon; Cincinnati, USA). Total operative time was 56 minutes with minimal blood loss and she tolerated the procedure well. Given her prematurity, she was admitted for postoperative monitoring and subsequently discharged home the following day. The repair has remained intact in follow up at 6 months.

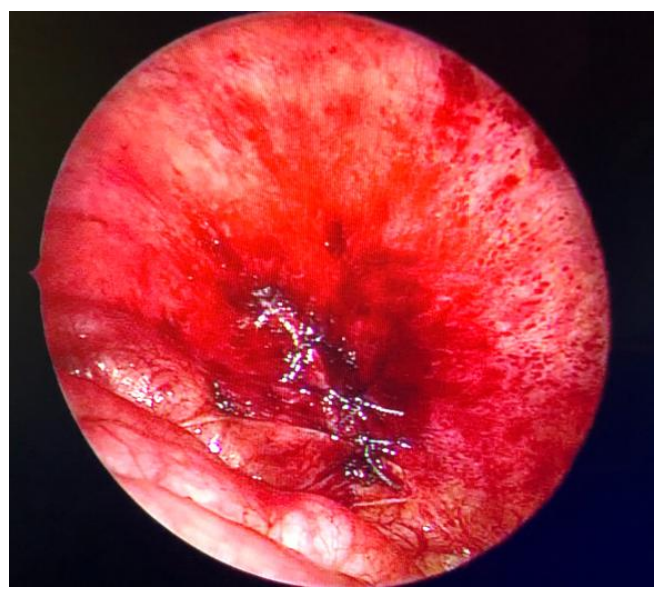

Figure 2 Intra-operative image following repair.

Given the rarity of CLH there is no clear consensus regarding the type and approach of repair i.e open vs. laparoscopic and primary vs. mesh reinforcement Lapa- roscopic approach to the repair of lumbar hernias has been successfully employed in adults and previous investigators have reported excellent visualization of the hernia contents and the edges of the fascial defects. Moreover, additional benefits of the minimally invasive approach are decreased pain, shortened length of hospital stay and better cosmetic outcome.[1]

This case of a laparoscopically repaired CLH suggests that laparoscopic approach is a feasible alternative to open repair.

\section{REFERENCES}

1. Arca MJ, Heniford BT, Pokorny R, Wilson MA, Mayes J, Gagner M. Laparoscopic repair of lumbar hernias. J Am Coll Surg. 1998; 187:147-52.

2. Carbonell AM, Kercher KW, Sigmon L, Matthews BD, Sing RF, Kneisl JS, et al. A novel technique of lumbar hernia repair using bone anchor fixation. Hernia. 2005; 9:22-5.

3. Touloukian R. The lumbocostovertebral syndrome: a single somatic defect. Surgery. 1972; 71:174-81

4. Rattan KN, Agarwal A, Dhiman A, Rattan A. Congenital lumbar hernia: A 15-year experience at a single tertiary centre. Int J Pediatr. 2016;2016. 